\title{
Proposing SMOCUNM - Security Mechanism for Offline Cloud User at Near line Mode
}

\author{
Rimmy Chuchra \\ Department of Computer \\ Science \& Engg \\ Sri Sai College of \\ Engg and Technology, \\ Manawala (Amritsar) \\ Punjab (India)
}

\author{
Rajwinder Kaur \\ Department of Computer \\ Science \& Engg \\ Sri Sai College of \\ Engg and Technology, \\ Manawala (Amritsar) \\ Punjab (India)
}

\author{
R. K Seth \\ Department of Physics \\ DAV University,Jalandhar
}

\author{
Kawaldeep Kaur \\ Department of \\ Computer Science \& Engg \\ Sri Sai College of \\ Engg and Technology, \\ Manawala (Amritsar) \\ Punjab (India)
}

\author{
Jayant Shah Singh \\ Department of \\ Computer Science \& Engg \\ Guru Arjan Dev \\ Polytechnic College, \\ Dhariwal (Gurdaspur) \\ Punjab (India)
}

\begin{abstract}
This paper discussed about different types of offline cloud technologies uses in different types of offline cloud services. Authors designed a new methodology named "SMOCUNM" (Security Mechanism for offline cloud user at near line mode) for implementing the security feature at Near Line mode in offline cloud services. Near Line mode actually provides saved data on system hard drive on on-going basis that cannot be accessed frequently. The motivation to utilize Near Line mode is it provides security on the time of connection reestablishment when offline cloud user comes back to online after completion of his or her task. The chances of viruses and threats may be reduced by utilizing this new designed methodology on the time of data uploading on the cloud server. The other benefits to utilize this Near Line mode for security purposes is its low cost, fast response and quick back up with unlimited storage access anywhere-anytime even in a matter of seconds.
\end{abstract}

\section{Keywords}

Offline cloud service, Offline cloud technology, online mode, offline mode, near line mode, security, cloud data storage.

\section{INTRODUCTION}

Offline cloud services pay high value or most popular now a days especially for those areas or regions where there is bad internet connectivity or you can say there is no internet connectivity [5].The reason to develop such kind of service by cloud service provider is to reduce the dependency of cloud users on the internet up to some extent and continue their task anywhere-anytime in the absence of internet connection [21]. For running any offline cloud service, an offline cloud web based application requires five basic core elements viz. CSS, DB, HTML5, Java Script and Manifest file. Each has their own function \& that can be shown in fig.1:

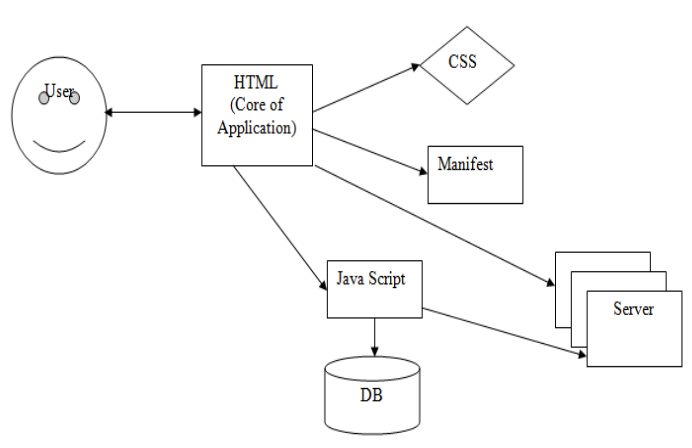

Fig.1:Core Elements of offline Cloud Web Application[28] [Accessed on-3dec2015].

- CSS: It describes how HTML page is rendered. Most importantly, it shows its default rendering behavior display.

- DB: Its main function is to provide local database storage.

- HTML5: It act as a core of the application used in offline access. Its main function is to provides cache that helps to calculate speed and reduce the load on servers.[15]

- Java script: its main function is to control the application.

- Manifest: Manifest is mandatory deployment descriptor component of an offline web applications-it simply lists all the files that need to be loaded.

As offline cloud user start working on any offline web application then at first, cloud user use the basic architecture of core elements as shown in fig. 1 then whole data is to be saved on system hard drive. Once the data is saved on the disk , the cloud user re-establish a connection. On the time of 
connection re-establishment the offline data is automatically uploaded on the cloud server by utilizing its distinct/unique feature of automated synchronization. This is a valuable automated tool for iCloud that offers automatic backup. It would be sure that this approach to work offline would not be dis-appear anytime in future. The major goal of offline cloud service is to reduce its support and maintenance cost as an example Fujitsu support [20] and 3D Experience support that works even on online mode but provides facility for offline access [25] where the list of different offline cloud services as shown in table in table 1 :

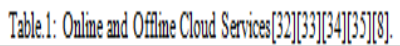

\begin{tabular}{|c|c|c|c|c|}
\hline S.10 & $\begin{array}{l}\text { Online Clould } \\
\text { Services }\end{array}$ & $\begin{array}{c}\text { Free Storage Space } \\
\text { Avalabitity }\end{array}$ & Offlue Cloud Services & $\begin{array}{c}\text { Free Stornge Sppace } \\
\text { Avalability }\end{array}$ \\
\hline 1 & Zip Cloud & $\cdot$ & Zinebox- Kind of cloud box. & . \\
\hline 2 & Noxy Stash- & $2 G B$ & $\begin{array}{l}\text { Google Mapss-Covers } 400 \\
\text { MB Area }\end{array}$ &. \\
\hline 3 & SpiderOals & SGB & Chrome Book & . \\
\hline 4 & AVGLivekire & $\overline{S G B}$ & Solidill orks 3D Experience & . \\
\hline j & Wuala by LaCie & SGB+Security & SOS.Suppototoflime Sertice & $\cdot$ \\
\hline 6 & Nettix Service & . & . & . \\
\hline 7 & Bullen Sertice & · & . &. \\
\hline 8 & SlimbBox Serice & · & - & $\cdot$ \\
\hline 9 & Vid) Neg Serice & $\cdot$ & . & . \\
\hline 10 & MPAFlie Sernice & $\cdot$ & $\cdot$ & . \\
\hline
\end{tabular}

The working of above discussed all cloud services is based on some older and some new offline cloud technologies that are listed in table 2:

\begin{tabular}{|c|c|c|}
\hline 5.10 & Older ofline storgege Techiniques & Nerr Offine Storage Techinioues \\
\hline ! & Cookies & Web Storage \\
\hline 2 & Plug In Based Storage & Teed SQLDatiabase \\
\hline 3 & Browser Spectic Features & Indexed Datbose \\
\hline 4 & Offine storgege in the era of HTMLS & Flle Storage \\
\hline
\end{tabular}

Correspondingly, Table 3 represents the list of several different cloud services with its free storage availability space:
Table3: Free Storage Space Availablity in Different Cloud Storage Services[13][16][28][30][31].

\begin{tabular}{|c|c|c|}
\hline S.No & $\begin{array}{l}\text { Name of the Cloud Storage } \\
\text { Services }\end{array}$ & FREE Cloud Storage Space \\
\hline 1 & Dropbox & Up to 25GB \\
\hline 2 & \begin{tabular}{|l|} 
Google Drive \\
\end{tabular} & $15 \mathrm{~GB}$ \\
\hline 3 & Box & 10GB \\
\hline 4 & Sky Drive & Up to 7 GB \\
\hline 5 & Sugar Sync & Up to $5 \mathrm{~GB}$ \\
\hline 6 & MEGA & Up to 50 GB Store files and 8GB Backup \\
\hline 7 & Tresorit & 3GB + End- to-End Encryption \\
\hline 8 & Pcloud & 750 GB+Provide orn ouline privacy \\
\hline 9 & Cubby & Up to 5 GB \\
\hline 10 & Ubuntu & Up to $5 \mathrm{~GB}$ \\
\hline 11 & \begin{tabular}{|l|} 
Version Cloud \\
\end{tabular} & New DropBox Type- + $500 \mathrm{MB}$ \\
\hline 12 & Zip Cloud & $\begin{array}{l}\text { Unlimitted Free Cloud Storage(upto 100 MB) AND ATTACH FILES } \\
\text { UP TO } 300 \mathrm{MB}\end{array}$ \\
\hline 13 & Gmail & Upto $15 \mathrm{~GB}$ and Email files up to $10 \mathrm{~GB}$ \\
\hline 14 & Office 365 & Paid $\mathrm{No}^{* *} /$ and S15 Dollars for 30 Days \\
\hline 15 & Microsoft one Drive & $15 \mathrm{~GB}$ \\
\hline 16 & Apple iCloud Drive & $5 \mathrm{~GB}$ \\
\hline 17 & HightTail & $2 \mathrm{~GB}$ \\
\hline 18 & One Drive & $5 \mathrm{~GB}$ \\
\hline 19 & \begin{tabular}{|l} 
Amazon Cloud Drive \\
\end{tabular} & $\mathrm{No}^{88}$ \\
\hline 20 & Syncplicty & $2 \mathrm{~GB}$ \\
\hline \multirow[t]{2}{*}{21} & Limer notes & \\
\hline & & NOTFREE * \\
\hline
\end{tabular}

Each offline cloud technology has its own function that can be described in table 4 and table 5:

Table.4: list of Older Offline Technologies with its function[27].

\begin{tabular}{|l|l|l|}
\hline S.NO & Older Offline Technologies & Functions \\
\hline 1 & Cookies & Only to provide little amount of data storage \\
\hline 2 & Plug-In based Storage & $\begin{array}{l}\text { It provides extension named Google Gears that is } \\
\text { uncommon and right version for usage and gives } \\
\text { better qualitative results when accessing its } \\
\text { service }\end{array}$ \\
\hline 3 & Browser Based Specific Features & $\begin{array}{l}\text { Its working is same like Plug.in Based storage } \\
\text { but must of working with specific browser only }\end{array}$ \\
\hline 4 & Offline Storage in the Era of HTMLS & $\begin{array}{l}\text { Its main function is the right utilization of origins } \\
\text { of data storage as an example they uses the } \\
\text { fomula like } \mathrm{I} \text { N where N is the number of } \\
\text { distinct storage mechanisms. }\end{array}$ \\
\hline
\end{tabular}

Table.: list of Nerw Offlime Technologies with its function[27].

\begin{tabular}{|c|c|c|}
\hline S.NO & New Offline Technologies & Functions \\
\hline 1 & Web Storage & $\begin{array}{l}\text { Only applicable for string values and } \\
\text { corespondingly provides Searlization that } \\
\text { provides local and sesion storage. It is generally } \\
\text { used for security purposes and removed when } \\
\text { window is closed. }\end{array}$ \\
\hline 2 & Web SQL Database & $\begin{array}{l}\text { It's offline SQL Database and implemented in } \\
\text { SQLite. }\end{array}$ \\
\hline 3 & Indexed Database & $\begin{array}{l}\text { Its function is to provide standard mapping like } \\
\text { local stonge but having index with some certain } \\
\text { fields especially in game levels. Because of our } \\
\text { games cannot be moved from all games data } \\
\text { structures. }\end{array}$ \\
\hline 4 & \begin{tabular}{|l} 
File System API(Application Program \\
Interface)
\end{tabular} & $\begin{array}{l}\text { Its main function is to create file folder } \\
\text { hierarchies. }\end{array}$ \\
\hline
\end{tabular}

All these above discussed 5 new offline cloud technologies are implemented practically but some of the technologies are too tough and not easily implemendted by cloud service providers. The list of those technologies are listed below:

- Storing Binary data.

- Extracting Binary Resources. 
- Handling Simultaneous data Operations.

- $\quad$ Structuring File Data.

- By storing miscellaneous data.

- Ensuring Cross Browser Capability [27].

Take care, some of the implemented offline cloud services gives some additional benefits that's why professioanls prefer more:

- $\quad$ Less Risk [9].

- Automated Synchronization Feature. - That provides a facility of automatic backup and it is sure this feature would not be disappear anywhereanytime.

- Guest Operating System can be easily added during scheduling [6].

- File sharing at offline mode on simple mouse button clicking [4] as an example Google Gear is an extension of source browser that helps to create offline web apps for file sharing [24].

- $\quad$ Reduce support and maintenance cost [2].

- Flexible bandwidth usage help us for saving cache in cloud without effecting the quality of service that ultimately maximize the co-operation between cloud acceleration \& peer assistance [3].

Due to all of these great benefits of offline cloud service is too popular but correspondingly it has one major drawback that is documents are not edited at offline mode [23] even when cloud service providers work on offline service then it does not show any reduction in functionality [23] in FIREBASE [18] \& STROM platform [11][1]. Both platforms give good performance at offline mode. The important key point is not all windows support offline access facility as like windows 7. The main reason is missing of CSC Service. This is the only reason you cannot share it when you try to start, then surely get error [26]. Similarly in case of adobe creative cloud, cloud user will not access this service for long time [26] due to its limitations but if it is used with box.net application [10] [12] then it gives an additional facility to save separate files and folders at free of cost and correspondingly it provides automatic updates in iPad and iPhones to sync files [7] at offline mode. These files used at offline mode named as "M-Files". These files load fast that reduce bandwidth consumption that ultimately avoid version issues [22].

The main idea of this research paper is to provide security of offline cloud user at near line mode. The basic working of an offline cloud user can be easily shown in figure 2 :

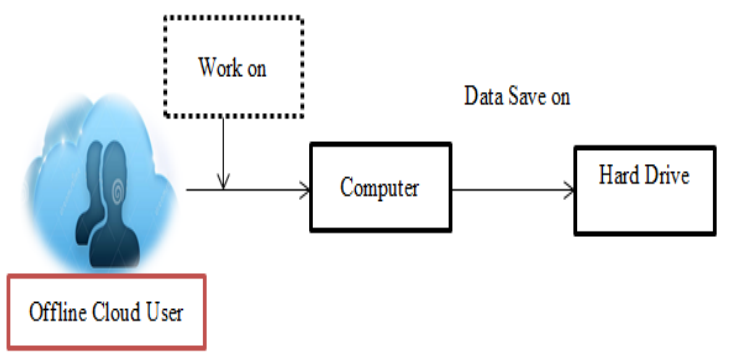

Once the data is saved successfully, by the offline cloud user then the whole content is transferred into system hard drive \& when user back to come online then the data is to be automatically uploaded on the cloud server by utilizing its distinct automatic synchronization feature. The main benefit to utilize this distinct feature is it will not be disappear anywhere, anytime. But in between the re-establishment of connection it may be possible the virus in the system may attach with confidential data that correspondingly ready for uploading on the cloud server. The author's contribution is to provide the security of offline cloud user at near line mode during re-establishment of connection. This mode defines as a compromise between the online and offline mode. The benefit to provide security at near line mode is to save human effort, time as well as cost before uploading data on cloud server and the other benefit is it provides unlimited storage, easy backup and an access anytime in a matter of seconds [17]. This near line mode is also fully integrated with google and other cloud storage services that does not need to adopt new programming model or data manipulation methods to store and retrieve data from near line storage [14]. As study completed by the authors they noticed the mission of google is how much time is saved while accessing offline cloud services as like time import and export. The main limitation of near line mode is the recently uploaded data will not be frequently used [19]. It declares the readable version at near line (that is you can only view or read the data but not download form the cloud server). Authors must be take care the data uploaded on the cloud server must be error-free especially on the time of data uploading on cloud server. If cloud user has some doubt of error then can applies different security algorithms for the prevention of the data. So, by utilizing this new designed methodology named "SMOCUNM" authors can easily provide security at near line mode on the time of connection re-establishment. The main benefit is it provides prior and early precautions to the offline cloud user from the different types of viruses, worms and threads. In addition, it automatically reduces human effort by applying various automated cloud testing mechanisms once the security at near line mode will be provided. Hence, authors say this ultimately improves user experience at offline mode [3]. So researchers suggest while working on offline mode cloud service providers may try to enable offline mode document editing option will be implemented as soon as possible.

\section{A ROADMAP- DATA MOVEMENT BY OFFLINE CLOUD USER}

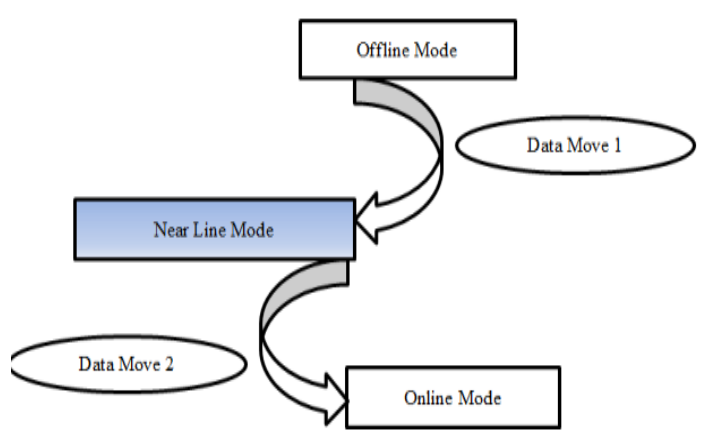

Fig. 3: Data Moves from Offline Mode to Online Mode.

Fig.2: Woking of Offline Cloud user 


\section{GRAPHICAL REPRESENTATION FROM O FF (OFFLINE) TO O N (ONLINE) SERVICE}

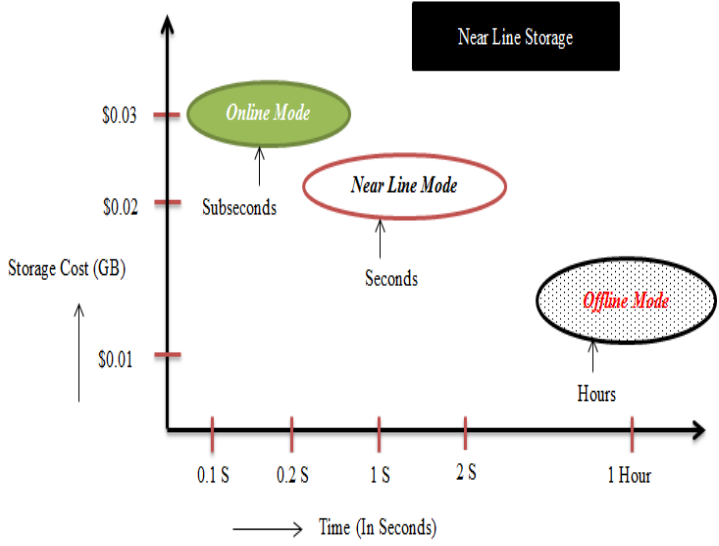

Fig.4: Movement of User- From Offline to Online[17][28][29] [Accessed on 3/12/2015].

Movement of data from offline to online mode:

$$
\begin{aligned}
& \text { Equation 1: } \mathrm{O}_{\mathrm{ff}} \rightarrow \mathrm{N}_{\mathrm{e}} \\
& \text { Equation 2: } \mathrm{N}_{\mathrm{e}} \rightarrow \mathrm{O}_{\mathrm{n}} \\
& \text { From Eq.1, Eq.2. } \\
& \text { Hence, } \mathrm{O}_{\mathrm{ff}} \rightarrow \mathrm{O}_{\mathrm{n} .}
\end{aligned}
$$

\begin{tabular}{|c|c|}
\hline & \\
\hline Olif & OHillin: \\
\hline Ii & Merime \\
\hline OII & Qulin: \\
\hline
\end{tabular}

The most important key point of offline cloud user at offline mode is when the data is saved once of system hard drive then transfer into cloud server and at last cloud user must have need to come online once again at once for successful updating. Hence, researchers said the complete dependency of offline cloud user is not eliminated till now.

\section{RESEARCH DESIGN}

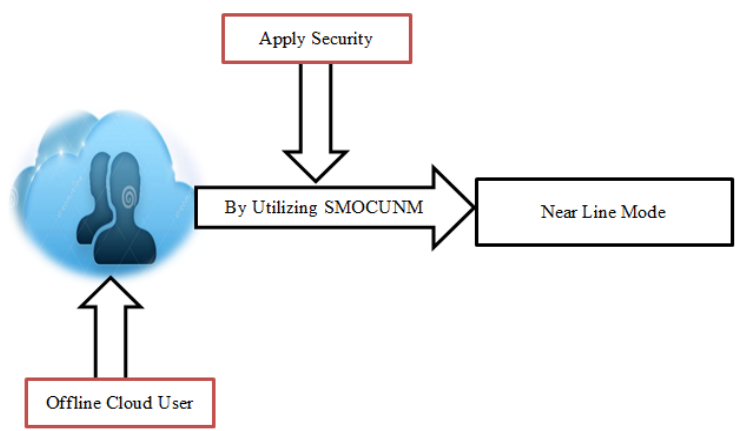

Fig. 5: Interaction of Oflince Cloud User with NearLine Mode through SMOCUNM.

\section{PROPOSED ALGORITHM- STEPS (SMOCUNM)}

Steps for SMOCUNM (On, Ne, Ws, Ds, V, OCU, Du)

Steps for SMOCUNM (On, Ne, Ws, Ds, V, OCU, Du)
\begin{tabular}{|l|l|}
\hline Table. 7: Nomenclature for SMOCUNM \\
\hline On & Online Mode \\
\hline $\mathrm{Ne}$ & Near Line Mode \\
\hline Ws & Work Status \\
\hline Ds & Data Status \\
\hline V & VIRUS \\
\hline OffCU & Offline Cloud User \\
\hline Du & Data Upload \\
\hline Dst & Data Storage \\
\hline HD & Hard Disk \\
\hline CS & Cloud Server \\
\hline
\end{tabular}

Step-1) Initialize OCU.

Step-2) CHK Ws of $\mathrm{O}_{\mathrm{ff}} \mathrm{CU}$. IF Ws: = TRUE Then $\mathrm{D}_{\mathrm{st}}:=\mathrm{HD}$ // Data is successfully stored on the system hard drive.

Step-3) IF $\left(\mathrm{O}_{\mathrm{ff}} \mathrm{CUWs}==0\right)$

\{

Ws $=$ Task Completed

\}

ELSE

Move to Step 2.

Step-4) Apply SMOCUNM for Checking the Presence of V. // Apply security at Near Line Mode on the time of re-establishment of Internet Connection.

Step-5) IF $(\mathrm{V}==0)$

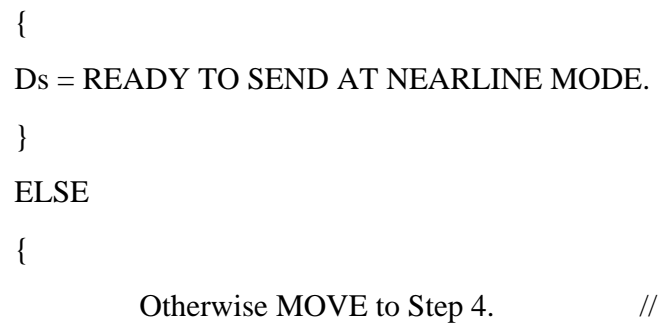

Otherwise MOVE to Step 4.

RECHECK the code once again.

$$
\text { \} }
$$

Step-6) AFTER THAT Ne Ds $\rightarrow$ Forward to On.

Step-7) HENCE, Du: = Cloud Server. // secured data is successfully send on cloud server.

Step-8) End. 


\section{COMMUNICATION FLOW METHODOLOGY: SMOCUNM}

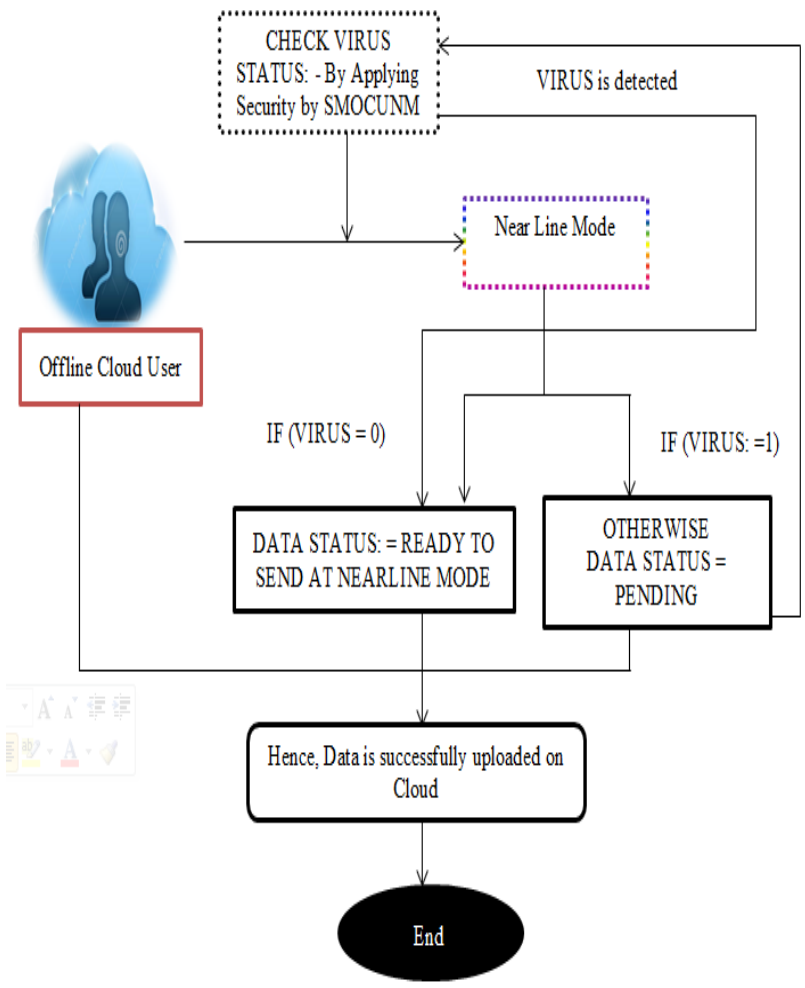

Fig. 6: Communication between Offline Cloud User and Cloud Server

\section{WORKING}

At first, as discussed in figure 2 when offline cloud user work on system at offline mode then the whole data is to be saved on system hard drive. In the $2^{\text {nd }}$ step, once data is successfully saved on system hard drive, after that offline cloud user try for re-establishment of connection for data uploading on cloud server. During the re-establishment of connection (it includes the time span when user send request to the browser till the internet is connected with the internet) this proposed methodology named "SMOCUNM" is applied for checking the presence of viruses and threats with successfully stored confidential data. At third step, if virus is detected by the SMOCUNM then once again sends back that specific confidential data to step 2 and again SMOCUNM is applied once more otherwise data forward to Near Line mode to online mode. Hence, at last step, that confidential data is successfully uploaded on the cloud server.

\section{CONCLUSIONS}

Different types of offline cloud technologies uses in different types of offline cloud services with its free storage space availability is discussed in this paper. Security at near line mode may be increased by applying new designed methodology named SMOCUNM (Security Mechanism for Offline Cloud User at Near Line Mode). The significance to design this new designed methodology is to provide protection of offline cloud user confidential data from different types of viruses, threats and worms when data resides at Near Line mode. The benefit to work with Near Line mode is it provides intermediate type of data storage that is actually not immediately available but can make online quickly without human intervention. In the end, authors said while utilizing this new designed methodology at Near Line mode authors easily provide protection of data from viruses and threats before uploading data on the cloud server ( or before re-establishment of connection when user back to online). Authors concluded things will be possible in future without internet connection. And the dependency of users on internet may be reduced up to some extent by utilizing offline cloud services.

\section{FUTURE SCOPE}

In future, the strength of security will be extended by providing more tighten security block that will be separately implemented on the platform of offline cloud technology. The parameters considerations taken by authors while designing the algorithm will be important factor.

\section{REFERENCES}

[1] Suyang \& oinghua Lu, October 2015. A Video cloud platform combining online \& offline cloud computing technologies, Journal personnel \& ubiquitous computing, Springer-Verleg, UK.

[2] Sen A, Rotta Mo, May-June 2015. Offline Risk Assessment of Cloud Service Providers, Cloud Computing, IEEE.

[3] Yuchun Guo, Changjia Chen \& Yingying Xu, October 2012. Offline Downloading: A Non-Traditional Cloud accelerated \& peer assisted content distributed Service, IEEE Explore: Cyber Enabled Distributed Computing \& Knowledge Discovery, Sanya.

[4] Kumar Rajesh \& Chachapara Kajal, 2015. Framework to establish offline file sharing in application as a service layer in cloud computing, International Journal of knowledge sharing platform.

[5] AB Erisson \& Olsson Magnus, 2011. A brand new offline web identifying and mitigating hurdles for developers, Workshop: On the future of offline web applications, USA.

[6] Jin Hai, Lu Zhiqiang,He Ligang \& Shao Zhiyuan, 2013. VSA: An offline Scheduling analyzer for Xen Virtual Machine Monitor, Future Generation Computer Systems, ELSEVIER.

[7] https://movilizer.com/technology/capabilitiesfeatures/online-offline.html.

[8] www.notebookreview.com/how/to/watch-onlinestreaming-videos-offline/.

[9] www.showingsuite.com/cloud-technology-for-realators/.

[10] mashable.com/201008/104box-offlinefiles1\#mqiuktmtxaqQ.

[11] www.edbrill.com/ebrill.nsf/dx/offline-mail-in-thebrowser.

[12] https://cloud.google.com/storage/docs/offline-mediaimport-export?hl=en.

[13] www.pcadvisor.co.uk/test-centre/internet/14-best-cloudstorage-service-2015-2016-UK-36142691/.

[14] www.eweek.com/storage/new-google-cloud-serviceoffers-online-data-storage-at-offline-prices.html.

[15] https://www.esoftload.info/offline-access-in-cloudapplications. 
[16] heavy.com/tech/2013/07/top-10-baest-cloud-storageapps-for-android-2013/.

[17] googlecloudplatform.blogspot.in/2015/03/introducinggoogle-cloud-storage-Nearline-near-online-data-at-anoffline-price.html.

[18] www.infoworld.com/article/2929853/mobiledevelopment/google-s-firebase-mobile-platformaccomodates-offline-usage.html.

[19] www.everbuying.net/product1097978.html.

[20] www.fujistu.com/globl/about/resources/news/pressreleases/2014/0514-02.html.

[21] www.mobilize.com/wp/wp-content/uploads/2014/02/wphow-do-you-use-the-cloud-without-an-internetconnection.pdf.

[22] www.m-files.com/en/document-management-mobile.

[23] pivotpoint.io/en-us/article/google-moves-docs-googledrive-offline\#.VKOJvU8rdkg.

[24] https://develpoer.salesforce.com/page/building-offlinesalesforce-applications-with-google-gears-and-Googleweb-Toolkit.

[25] blogs.solidworks.com/solidworksblog/2015/03/usingthe-cloud-offline.html.

[26] www.puryear[it.com/blog/2012/11/29/windows-7offline-files-does-not-work-offline-files-servicecscservice-dies/.
[27] www.html5rocks.com/en/tutorials/offline/whats-offline/.

[28] www.ibm.com/developerworks/library/wa-offlineweb/.

[29] https://www.google.co.in/search?hl=en\&site=imghp\&tb $\mathrm{m}=\mathrm{isch} \&$ source $=\mathrm{hp} \& \mathrm{biw}=1366 \& \mathrm{bih}=667 \& \mathrm{q}=\mathrm{ONLINE}+$ OFFLINE+NEARLINE+IMG\&oq=ONLINE+OFFLINE +NEARLINE+IMG\&gs_l=img.3...678.5588.0.5843.27.1 6.0.0.0.0.514.514.5-

1.1.0.......1ac.1.64.img..26.1.513.POZNu_zNGqk\#imgrc $=3 \mathrm{tQyXFkyro} 4 \mathrm{aNM} \% 3 \mathrm{~A}$.

[30] http://in.pcmag.com/storage/40065/feature/the-bestcloud-storage-services-for-2015.

[31] http:.//www.cnet.com/how-to/onedrive-dropbox-googledrive-and-box-which-cloud-storage-service-is-right-foryou

[32] http://www.pcworld.com/article/2453999/chromebooksbeyond-the-cloud-everything-chromebooks-can-dooffline.html.

[33] http://blogs.solidworks.com/solidworksblog/2015/03/usi ng-the-cloud-offline.html.

[34] http://www.spotpos.com/documents/sos.pdf.

[35] http://www.theverge.com/2012/4/24/2954960/googledrive-dropbox-skydrive-sugarsync-cloud-storagecompetition 